\title{
WHEN WORDS COLLIDE: EL INGLÉS Y EL HUMOR EN TRES TRISTES TIGRES
}

Maria Jesús Rodríguez Medina

Universidad de Las Palmas de Gran Canaria

RESUMEN

Para los profesionales de la ironía, la parodia y el humor, el uso expresivo e intencionado de anglicismos y el cambio de código (mezcla en el discurso de las lenguas inglesa y española) es una forma muy eficaz de captar la atención de los hablantes. Muestras de ello son algunas de las obras de escritores como Díaz Valcárcel y Muñoz Molina, en las que el inglés cobra un protagonismo especial. Sin embargo, en ninguna de ellas llega esta tendencia al nivel de Tres tristes tigres, de Guillermo Cabrera Infante, donde la aparición frecuente de anglicismos y los cambios constantes de código son la fuente principal de los controvertidos juegos de palabras del autor y la base fundamental de su estilo. En este artículo, realizamos un análisis de las distintas funciones expresivas del inglés en la obra, entre las que destacan la parodia de la jerga nocturna habanera y la búsqueda de la comicidad.

\section{INTRODUCCIÓN}

En los últimos años, cada vez vez es más frecuente que los profesionales españoles de la ironía y el humor recurran al inglés para aumentar la originalidad y comicidad de sus creaciones. Tanto en las columnas y artículos de prensa más leídos como en los programas de radio y televisión de mayor audiencia, el uso expresivo e intencionado de anglicismos y cambios de código (mezcla en el discurso de las lenguas inglesa y española) se ha convertido en una forma muy eficaz de llamar la atención del público (Rodríguez Segura 1999; Rodríguez Medina 1999, 2000). De hecho, hay que aclarar que 
no se trata de una característica reciente y exclusiva del lenguaje periodístico, sino que ha sido un ardid muy recurrente en la literatura contemporánea, en especial en el ámbito hispanoamericano, donde la cultura estadounidense también ha dejado una huella notable en gran parte de la producción literaria.

En la novela Mi mamá me ama (1988), el puertorriqueño Díaz Valcárcel emplea el inglés en sus parodias para satirizar los prejuicios linguísticos de las clases altas de Puerto Rico y los conflictos sociopolíticos que se originan por la confrontación del mundo hispano y anglófono en este país. Este recurso, denominado por Ullmann efecto evocador secundario, consiste en «la acumulación de palabras inglesas, o incluso sintagmas y frases, que refleja una clara actitud de crítica y sátira del esnobismo" (Gómez Capuz, 1997: 88). En este sentido, nos parecen muy esclarecedores los siguientes fragmentos de la novela:

[...] recuerdo que inesperadamente apareció su mamá y fue hasta el medio de la pista donde bailaba un soul apretadito con Lucy Foreman y lo abrazó y le dijo cómo te sientes hijito querido este week-end tan largo y alejado del regazo materno. Ted tuvo una reacción irracional: se bajó los jeans y le enseñó el trasero [...].

Los hijos chiquitos de la pobre Altagracia se quedan mirando como tontos la chimenea de la primera planta, con sus leños traídos de Vermont [...]. La usamos cuando nos visita algún congresista o mi padrino el licenciado Julio Borrero Blanco, socio de papá. Cuando [...] nos visitan papá pone el aire acondicionado central a todo dar hasta que el frío se hace verdaderamente fuerte; entonces todos nos ponemos suéters; papá se sienta en su butaca junto a mamá y los invitados, con la bufanda azul y blanca alrededor del cuello y los pies calzados en zapatillas comodísimas; entonces todos nos ponemos suéters [...]. Con la bufanda suelta sobre el pecho, mi padrino se frota las manos heladas ante el fuego, fumando su pipa, comentando en inglés la crudeza del tiempo, el bajón de la tempertura [...]. Y papá dice que esa temperatura, la chimenea, la pipa, ese estilo de vida en fin nos ayuda a parecernos más y más a nuestros conciudadanos del Norte [...]. Esas reuniones las celebramos casi siempre en verano [...]. En navidad utilizamos la chimenea para propósitos más hermosos y desprendidos. Los hijos de los socios de papá colocan largas medias junto a la chimenea en christmas, y entonces papá hace descender por el interior de ella a un Santa Claus cargado de juguetes (amamos la tradición) ${ }^{1}$.

En España, llama la atención el caso de Muñoz Molina, que cuenta con una novela corta, Carlota Fainberg (1999), donde la presencia constante e intencionada de anglicismos se convierte en la base fundamental de su prosa crítica y satírica, que pone en evidencia, de forma muy sutil, el funcionamiento de los departamentos de español en Estados Unidos ${ }^{2}$ y el proceso de transculturación que sufren algunos de sus miembros:

' Si bien en el primer ejemplo el autor usa anglicismos puros con fines paródicos (week-end, jeans), en el segundo, son los anglicismos culturales (Lorenzo 1991: 68), es decir, la copia de hábitos culturales de los países anglófonos, los que provocan la hilaridad del lector, ya que se describe cómo una familia rica de la isla recurre incluso al aire acondicionado para poder pasar frío como los estadounidenses (la chimenea sirve también para seguir las tradiciones navideñas a la americana).

${ }^{2}$ Llama la atención que, a medida que avanza la novela, el autor vaya disminuyendo la proporción de anglicismos empleados por el personaje, en una especie de redención irónica de su personalidad. 
[...] con los años me he acostumbrado a lo que al principio me atosigaba tanto, a las formalidades y reservas de la etiqueta académica norteamericana, ya que ya me siento incómodo, o más exactamente, embarrassed, ante cualquier despliegue excesivo de simpatía, que casi nunca llega sin su contrapartida de mala educación. (1999: 20)

[...] El texto es a la vez la batalla y el botín, o, para usar la equivalencia valientemente sugerida por Daniella Marshall Norris, todo semantic field es en realidad un battlefield, inluso, se me ocurre a mí [...] un oilfield en el que la prospección petrolífera sólo tiene éxito verdadero cuando llega a las capas más profundas. (1999: 37)

Pero, sin duda, Cabrera Infante es el máximo exponente de los escritores de habla hispana que gustan de utilizar el anglicismo como medio expresivo en sus escritos. Una muestra clara de ello es su libro Tres tristes tigres $\left(1967^{3}\right)$, donde no sólo para imitar la jerga y el ambiente anglicados de la noche cubana ${ }^{4}$ de una época determinada, sino también con la intención de buscar formas novedosas de expresión, los anglicismos y el cambio de código se convierten en un filón muy productivo como recurso para lograr todo tipo de efectos estilísticos ${ }^{5}$, explotar al máximo las posibilidades del idioma y lograr la sonoridad de sus conocidos juegos de palabras ${ }^{6}$ o, como los llama Varanini (2003), delirios verbales, en los que siempre están presentes la ironía, la parodia y el humor.

\section{ANÁLISIS DEL USO DEL INGLÉS EN TRES TRISTES TIGRES}

Antes de adentrarnos en el análisis, hemos de destacar el carácter fragmentario y aparentemente caótico tanto de la estructura de la obra como del lenguaje empleado. Se trata de una novela de voces narrativas que Rodríguez Monegal (1969: 38) define como «collage de textos hablados deliberadamente no sucesivos» y de la que destaca «la facilidad del autor y de sus personajes para dividir una palabra en sus componentes fonéticos o gráficos, para dar la vuelta a una frase [...], esa capacidad infinita de hacer variaciones sobres sus silabas o significados».

Aunque nos referimos a una habilidad experimentada ya en el siglo XVIII por Laurence Sterne (The Life and Adventures of Tristram Shandy), y practicada posteriormente por otros muchos autores (cf. nota 6), la aportación de Cabrera Infante ${ }^{7}$, lo que ofre-

${ }^{3}$ Aclaramos que la edición que hemos manejado es la de 1991.

${ }^{4}$ Uno de los mayores logros de esta novela es su capacidad para transmitir, en el lenguaje escrito, el ritmo y la calidad de la lengua hablada. En este sentido, la influencia de Mark Twain en Cabrera Infante es evidente (Rodríguez Monegal 1969:43).

${ }^{5}$ Los anglicismos que explota Cabrera Infante crean efectos de vistosidad por el «atractivo del lexema exógeno» (Gómez Capuz 1997: 668) y el ritmo atípico de formas fónicas inusuales en español.

${ }^{6}$ Preguntado acerca de qué es la literatura (Hernández 2001), el propio Cabrera Infante reconoce que le parece «un vasto campo de juego» y que ha llevado el lenguaje cubano y este juego «a extremos que nadie había hecho antes en español, tal vez a excepción de Gómez de la Serna, con los antecedentes, en francés, de Jarry, Satie y Queneau y, en inglés, de Lewis Carroll y Joyce». Según Aguilar (2000:9), «su tema no es tanto la memoria, sino el modo en que ésta se despliega en la literatura y en el cine y es desde esta perspectiva que hay que entender su obsesión por los juegos de palabras».

${ }^{7}$ El cubano ha recibido muchas críticas y comentarios desdeñosos de otros literatos, estudiosos y lectores, quienes, contrarios a esta característica tan marcada de su estilo, se muestran molestos por la constante deformación de la lengua exhibida en sus obras (Grosso 1968: 29). 
ce de nuevo su prosa, es la utilización de una segunda lengua - en este caso es el inglés - que entra a formar parte de la complicada maraña de manipulaciones linguísticas que constituyen el eje de los diálogos. No obstante, hemos observado que, a pesar del caos aparente, los casos recogidos ${ }^{8}$ responden a usos claramente intencionados y diferenciados que - si bien es cierto que en ocasiones se solapan - se pueden distinguir u ordenar por su función en el discurso:

\subsection{Parodia del habla anglizada}

En este apartado se incluyen las palabras y expresiones inglesas que aparecen transcritas según la fonética de los hablantes de la noche habanera. De esta forma, Cabrera Infante consigue transmitir, con gran viveza, las coletillas en inglés típicas de los que frecuentan los ambientes descritos en la novela:

- Fue el chif el que hizo presión con dos ministros que son socios fundadores y tuvieron que admitirlo así como tú lo-o-yes. (p. 43)

- Parecía dominar absolutamente el chowcito ${ }^{9}-\mathrm{y}$ ahora tengo que explicar qué es el chowcito. El chowcito es el grupo de gente que se reunía a descargar en la barra, pegados a la vitrola, después que terminaba el último show. (p. 63)

- Me dijo, no están: se fueron a la playa, de wikén, sube que vamos a estar solos. (p. 70)

- A las niñas del Yacht no le hace falta tocar el bongó. - Yo soy una niña del Yat-dijo. (p. 99)

- Y me escapo allá al San Yon o a la Gruta o donde me da la gana entre show y show y es eso lo que estoy haciendo ahora, yonderstán? (p. 273)

- Ahora no voy por los naicluses, como decía La Estrella, porque [...] (p. 281)

\subsection{Anglicismos y pseudoanglicismos ${ }^{10}$ humorísticos}

El autor recurre con frecuencia a anglicismos y pseudoanglicismos, con los que persigue aumentar la comicidad de las situaciones donde aparecen:

- El punto final de la trompada esponjosa al estropeado punching-bag que debía tener todo su mecanismo punch-drunk ${ }^{11}$ hace rato. (p. 39)

- Y los panes con bisteques que hay casi en la esquina, en un puesto ad hoc $\operatorname{dog}^{12}$. (p. 40)

- Pensé en aquella mulata enorme [...] cantando en el Saint-John (ahora todos los niteclubs de La Habana tenían nombre de santos exóticos: ¿era sisma o snobismo?) (p. 114)

\footnotetext{
${ }^{8}$ Aunque el autor inserta los anglicismos en el texto sin rasgos tipográficos distintivos, preferimos utilizar la cursiva para que la localización de los distintos ejemplos sea más sencilla.

${ }^{9}$ Obsérvese lo curioso e hilarante que resulta el diminutivo de este anglicismo parcialmente asimilado.

${ }^{10}$ Nos referimos al léxico de apariencia inglesa que, en realidad, no existe en esta lengua.

11 «Demonstrating or characteristic of the behaviour of a person who has suffered repeated blows to the head, esp. a professional boxer» (Collins 1991: 1.257).

${ }^{2}$ Híbrido de hot dog y la expresión latina ad hoc.
} 
- Baby, aquí será una modelo, pero en Nueva York o Elei sería una callgirl de lujo. (p. 155)

- Emce. Nota a pie: abreviatura fonetizada de Master of Ceremonies, maestro de ceremonias. (p. 180)

- No quería ser víctima de ninguna lynching mob, ya que yo parecía un extranjero que abusaba de un nativo indefenso. (p. 189)

- Insistió en llevarnos a ver otra clase de espectáculos (show). (p. 190)

- Ya íbarmos cantando las Variaciones Quistrisini (copyright, Boustrophedon Inc). (p. 212)

- Ya me estaba cansando de este dealer del recuerdo. (p. 307)

- Como los canales de esta Venecia del rico, donde los felices propietarios podrán guardar su automóvil en el cart-port y su lancha en el yacht-port. (p. 320)

- La muerte es la gran niveladora: la buldozer de Dios (p. 334)

- Voy al baño, vulgo pipi-room. (p. 347)

- ¿Sería un crítico literario in disguise? (p. 395)

- Que lo llamara y le dio su teléfono y lo repitió como un jingle. (p. 401)

- Permiso para un leve sobresalto. (Acento mexicano, please). (p. 417)

- ¿Qué fue primero el water-closet o el saloon? (p. 427)

- Hombre, por favor, tú eres un perro viejo. An old hound. Es más elegante. (p. 432)

- Le grité considérelo un placer mutuo, Lord Shit-Land. (p. 443)

\section{3. Cambio de código al inglés}

En la parte del libro con más diálogos, el cambio de código al inglés se convierte en un recurso constante y muy productivo, con el que Cabrera Infante logra creaciones humoristicas, satíricas y onomatopéyicas muy singulares, basadas en contrastes entre las dos lenguas. Apreciamos dos tendencias generales:

- La palabra o expresión española aparece seguida de su traducción inglesa o «versión» deformada en inglés:

- ...y supo que era Ella. Es decir, she. (p. 308)

- Ese es un mal ajedrecista o, como tú dices, a pool player. (p. 357)

- Un jugador de corazón, a chest-player, a jazz-player. (p. 357)

- Que demostraba (to demons trate, where demons fear to trate) que Ardébol [...] (p. 363)

- Soy el rey del disimulo. Actor at large. (p. 348)

- ¿Quién?-me dijo--. ¿Alma Mahler Gropius Werfel? Spermaceti. Sperm-whale. Vallena de esperma. Whale? I mean, where? Ahoy! Ahoy! On starboard, sir. A stribor. (p. 364)

- Estoy dispuesto a llegar donde el cepillo no toca. Where brushes fear to sweep. Mierda, doblan por Quince. La calle Quince, perdón Bertrand. Where Russells fear to think. Se nos van (p. 364)

- Incluyendo esta bitácora o log, el $\log$ de Gog y Magog.

- Colisión de sexos. Coalición. When works collide. When words collide. Les habla. (p. 365)

- Ojalá todas las mujeres tuvieran una sola cabeza (maiden-head). Cuéligula. (p. 372)

- I laughed. Digo, me reí. (p. 374) 
- Tú eres el Memorión, ¿recuerdas? Mr. Memory. Memory Blame. (p. 388)

- Pensaba que me dejarías en la boca del metro, subway, tube o subte. (p. 404)

- ¿Por qué tus preguntas viene en tres? Everything happens in trees, diría Tarzán. (p. 425)

- Me volvía a quedar durmiendo dreamiendo soñando con los leones marinos de la página ciento uno: morsas: morcillas: sea-morsels. Tradittori. (p. 445)

- El pensamiento fluye en los dos idiomas, es decir, se produce un auténtico cambio de código en el que se entremezclan el español y el inglés, con mayor presencia del primero:

- [...] halagando a Silvestre: a mystic bond of writerhood unía a esos dos. (p. 214)

- ...y la estranguló de esta suerte (What the hell are you doing you a sex maniac or what oughh oughh). (p. 309)

- Happy-happy de Ulacia las veces en que la vida copia al arte. (p. 334)

- Se sonrió. Se rió. Se puso serio. One two three. Estuvo callado un rato. (p. 346)

- No te preocupes. Estás en la inercia del pobre. Eso cambiará y pronto. What are you? A sooth-sayer? (p. 351)

- ¿No hay nada sagrado para ti? Have you no honor? (p. 354)

- Podíamos muy bien ser Damón y Fitias. Who's who? Elige tú que canto yo. (p. 361)

- Que se llama lda y cada Ida no tiene su Venida? Harping in the dark. ¿Ina? (p. 363)

- Trabajaré en la Ionescu. Un momento. Have you no honor? No country? No loyalty to royalty - royalty to loyalty? Esta Magalena no es tan niña, la otra es tampoco tan vieja. One at a time. Cábala. No estadísticas. Cuban bodice. Cuban boy. Cuban body. Body by Fischer. Magele Wash Ramper, se exhibe en La Rampa. Ambar Motors. Sepia Motors. Sexual Motor. General Motels. Fordnicando. (p. 370)

- Yeah? Sheriff Silver Star, we're running outa gas. Afectaba un acento tejano. Ahora era un marshal del oeste. $O$ cheriff adjoint. (p. 373)

- El Singular, Top Banana [...]. Yo, el Dos, el Yang de tu Yin, Eng de tu Chan, el Gran Paso, el Discípulo, Number Two, Second Banana. (p. 405)

- Conozco una o dos que no son locas. More power to you. Pero redúcete a tus cuarteles. (p. 412)

- No has salido todavía de entre los muertos. That's what you think. ¿Qué eres entonces? (p. 430)

- Laura, Laura Elena, Laura Elena Día. Never heard of her. Laura Día. (p. 434)

\subsection{Cambio de código al inglés y otras lenguas}

Cabrera Infante no se ciñe solamente al inglés, sino que enriquece también los diálogos con el cambio de código a otros idiomas, sobre todo el francés, aunque también incluye el italiano, el alemán y hasta el latín:

- Ca alors - me dijo-. Cheri, viens. Embrassez-moi - eso se lo dijo a Arsenio Cué, por supuesto.

- Oh, dear-dijo Cué y se olvidó de nosotros todos para hundirse en aquellos cúbitos, en aquellos radios, en aquellas clavículas bilingües. Trilingües. (p. 96) 
- ¿A dónde? - dijo la novia de Cué.

- Yes where? - dijo Cué.

- Al pipi-room, cherís ${ }^{13}$-dijo Vivian. (p. 96)

- Estás más muerto que el Zar: Kaputt tot, dead, difunto mandado pal otro mundo, ñampiado, mort, morto profundo ${ }^{14}$. (p. 253)

- Ah, viejito, es mejor que Offenbach, te lo juro, porque está here, hier, ici, aquí en esta tristeza habanera y no en una alegría parisién. (p. 296)

- ¿Qué pasa? What am I? A jester? A poor player. A pool player [...]. Estamos arrancados. Kaputt. Fini. Broken. Nos pelaron. (p. 351)

- Ensayos, essais, essays. (p. 362)

- El refranero a la lanterne rouge. Red Light District. (p. 362)

- Seguro con una amiga. Le amiche. The tits of lovelyness. Bixfocales. (p. 364)

- Se empieza a hablar de Cuésimodo. Le Tout Paris le tutea. Le dicen Cuési. Algunos le Ilaman, americanizados, Mody. Son los que dicen The Bastill cuando pasan una noche en el precinto (también anglicismo) o un drink of hydrohoney y bailan country-dances, adelantados a su tiempo. Quel horreur le Franglais. La culpa es de estos Plantagenet con sus idas y vueltas. Les anglais a la lantern! We shall take care of the latch, Joan of Arc. Cuésimodo repite el viaje y la elección. (p. 366)

- Va hoy al Equus Insanus taverne. Quel horreur le Franlatin. (p. 366)

- Claro, por supuesto, of course, bien sure, natürlich. (p. 399)

- Crees, cabrón, en el padre Hugo que está en el Olimpio et dans le tout Parnasse? Never heard of them. (p. 429)

- Me cago en Némesis. To defecate. Era un francés. Luego se rí́ y dijo le cabrón est sorti meme sous la pluie. (p. 430)

\subsection{Referencias cinematográficas}

De todos es conocida la afición del escritor al séptimo arte, por lo que no es de extrañar que inserte en el texto referencias continuas en inglés al mundo del cine:

- Veo en big close-up su mano en el bolsillo. (p. 166)

- Temí una situación de slapstick si agarraba el bastón. (p. 178, p. 193)

- [...] en el carro de Cué que se desliza como un travelling del castillito de la La Chorrera a los frontones. (p. 293)

- A veces pienso que puede ser el comienzo de un fade-out. (p. 303)

- Se oyen en off voces de Preparen Apunten ¡Fuego! (p. 307)

- Esta es una versión boy-meets girl/boy loses girl/boy finds girl de la vida (p. 335)

- Y en el mismo shot del recuerdo veo a mi tío. (p. 336)

- Andaba como el Mad Doctor ${ }^{15}$ por el patio. (p. 337)

- Había que oír a Arsenio Cué y también ver la cara de las damas de compañía. The Greatest Show in Hearse ${ }^{16}$. (p. 375)

${ }_{13}^{13}$ Españolización de cheris.

${ }^{14}$ Insertado en una elegía que cuenta incluso con rima.

${ }^{15}$ En español, El profesor chiflado.

${ }^{16}$ El mayor espectáculo del mundo. 
- Llegaron los body-snatchers ${ }^{17}$ y le pusieron [...] (p. 400)

- Hubiera sido un background Universal Pictures para mi diálogo con Prieta Dubois. (p. 405)

- ¿Titulado como, Desenmascarado o Evilly the Kid Strikes Back ${ }^{18}$ (p. 429)

- He went away singing in the rain ${ }^{19}$. Nos reímos. (p. 430)

\subsection{Intertextualidad con referencias culturales del ámbito anglófono}

Se trata del uso de nombres, frases o situaciones de personajes históricos o literarios del mundo anglosajón que intercala en el texto:

- Claro que perdí la batalla. My Kingdom for a $\operatorname{cow}^{20}$ (p. 327)

- Ah Oscarwilderness ${ }^{21}$. (p. 327)

- Alice on Yonderland. Alice in Underlandia. Aliciing in Vomitland ${ }^{22}$. (p. 350)

- Levanta el perfil Ezra Pound-quake ${ }^{23}$ y su voz trémola llena el carro. (p. 375)

- ¿Pel-ly Mason ${ }^{24}$ conocel caso? By heart. Mason es un contradictorio. (p. 407)

\subsection{Manipulación humorística de los nombres de los personajes}

El autor experimenta también con los nombres de los personajes, que va adecuando a la situación en la que se encuentran. Para ello, suele valerse de recursos intralinguiísticos del español, pero en ocasiones echa mano también del inglés:

- [...] vamos a cocomer, dijo imitando un gago este Bustrófunny-man. (p. 208)

- [...] y las quejas de Yugazbilly the Kid. (p. 256)

- Tú. Silvestre the First. (p. 404)

\subsection{Anglicismos sin valor expresivo}

Por último, reproducimos aquellos anglicismos que no responden, en realidad, a ningún fin estilístico o expresivo. Resultan menos interesantes que los casos anteriores,

${ }^{17}$ Los ladrones de cuerpos.

${ }^{18}$ Billy el niño.

${ }^{19}$ Cantando bajo la lluvia.

${ }^{20}$ Recuérdese la frase original de Ricardo III My kingdom for a horse!

${ }^{21}$ Alusión clara a Oscar Wilde, cuyo apellido convierte ingeniosamente en wildemess, que significa «soledad, desierto, yermo».

${ }^{22}$ Alicia en el país de las maravillas se presta en inglés, como vemos, a cambios jugosos. Lewis Carroll es uno de los escritores que más han influido en Cabrera Infante (cf. cita 6), por lo que referencias de este tipo eran de esperar en la novela.

${ }^{23}$ En este caso juega con el nombre del escritor Ezra Pound, al que añade quake «temblar», seguido de voz trémola.

${ }^{24}$ Alude al abogado Perry Mason, protagonista de los libros de Erle Stanley Gardner, conocido mundialmente por la serie televisiva. 
ya que se trata simplemente de anglicismos puros que designan objetos o realidades a los que los hablantes de español, en especial los del ámbito hispanoamericano, se suelen referir en inglés:

- Vestía un pull-over blanco y shorts azul celeste y alpargatas de fantasía. (p. 56)

- Yo andaba siempre por los cabarets y nite-clubs y eso, haciendo fotografías (p. 61)

- Se dejaba llevar mientras fumaba un cigarro king-size en una boquilla negra. (p. 98)

- Esa que toca entre show y show y cuando termina el último show para que la gente baiJe (p. 91)

- [...] con el bastón que se le cayó tres veces en el corto hall del lobby. (p. 183)

- No me reí por lo de playboy internacional, sino por su falso disgusto. (p. 183)

- Cué saludó al barman o al dueño. (p. 321)

- Te burlabas de oír a Palestrina sentado en un jet. (p. 325)

- Es un blue-print dibujado con tinta simpática. (p. 331)

- Era una niña con shorts. (p. 353)

- ¿Qué éramos? ¿Clowns, el primero y el segundo? (p. 391)

- Porque la bolsa tendría zippers para abrir secciones. (p. 383)

- Ha estado bajo tratamiento. Intenso. Electroshocks. Mazorra no y por suerte. (p. 399)

- Fue como si tirara la toalla al ring [...]. Considéralo un $K O$ técnico. (p. 419)

En definitiva, hemos comprobado cómo Cabrera Infante, virtuoso también del inglés, saca el máximo partido a las posibilidades que le brinda este idioma en su búsqueda del goce estético a través de las palabras.

\section{BIBLIOGRAFIA}

Aguilar, Gonzalo (2000): «Trópico del infante», Revista Clarín, suplemento dominical del 12 de noviembre, 8-9.

Cabrera InFANTE, Guillermo (1991) [1967]: Tres tristes tigres, Barcelona, Seix Barral. Díaz VALCÁRCEL, Emilio (1988) [1981]: Mi mamá me ama, E.U.A, Editorial Cultural.

GómEz CAPUZ, Juan (1997): Anglicismos en español actual: su estudio en el registro coloquial, Tesis doctoral, València, Universitat de València.

Grosso, Carlos (1968): «Comentario de la novela Tres tristes tigres», El escarabajo de oro $35,29$.

HernÁndez Cuéllar, Jesús (2001): «Lo peor del dragón está en la cola. Entrevista con Guillermo Cabrera Infante», Contacto Magazine, (documento disponible en Internet, http://www. contactomagazine.com/infante.htm, publicado en formato papel el 27 de octubre de 2001).

LoREnzo, Emilio (1991): «Anglicismos y traducciones», Studia Patricia Shaw Oblata II, Oviedo, 67-79.

Rodríguez Medina, María Jesús (1999): Los anglicismos sintácticos: estudio de dos manuales de informática traducidos, Tesina inédita, Las Palmas de Gran Canaria: Universidad de Las Palmas de Gran Canaria

- (2000): Los anglicismos de frecuencia sintácticos en los manuales de informática traducidos, Tesis doctoral, Las Palmas de Gran Canaria, Universidad de Las Palmas de Gran Canaria. 
RodRíGueZ MONEGAL, Emir (1969): «Estructura y significaciones de Tres tristes tigres», Sur 320, 38-51.

Rodríguez Segura, Delia (1999): Panorama del anglicismo en español, Almería, Servicio de Publicaciones de la Universidad de Almería.

SinClaIR. J. et al. (1991): Collins English Dictionary, Glasgow, HarperCollins.

VARANINI, Francesco (2003): «Guillermo Cabrera Infante», Periodico non apologetico di letteratura, documento disponible en Internet, http://www.eseresi.it 